\title{
EL PROCESO POLÍTICO EN CUBA Y LA CUESTIÓN DE LA REFORMA
}

\author{
Anthony Kapcia *
}

Una realidad incontrovertible de la Cuba de hoy es que, a partir del año clave de 1989, cuando se inició el derrumbe alumbrador del bloque soviético, se ha visto en la isla un proceso de transformación sin precedentes, o sea en la economía, en la sociedad, en el contexto político e incluso en aspectos de la misma ideología. Tanto en la literatura académica como en los medios masivos de comunicación, se suele encontrar una gama bastante extensa de juicios y opiniones distintos acerca de la naturaleza del dicho proceso de cambio, diferencias que, como en el período 1959-1963, reflejan la polarización intensa de perspectivas ideológicas acerca de la Revolución ${ }^{1}$.

A pesar de este debate, sin embargo, resulta posible identificar algunos aspectos evidentes de consenso sobre el proceso. En primer lugar, por ejemplo, no se cuestiona por lo general la existencia en Cuba de un proceso de reforma económica, aunque sí se debate mucho la naturaleza y los resultados de tal proceso. Así es posible afirmar que, entre la comunidad variada de los que observan a Cuba desde fuera, la evolución de una nueva economía cubana es ya una realidad bien establecida. Igualmente, parece haber consenso similar acerca de la inevitabilidad de que este proceso, que ha seguido la crisis tan profunda y tan desestabilizadora desde $1989^{2}$, afecte muchos aspectos de la sociedad cubana. Otra vez, se enfoca el debate a los efectos precisos y fundamentales de tal proceso, pero no se cuestiona la realidad de la evolución accidental de una nueva sociedad cubana que por fin emergirá de las crisis y los cambios.

No obstante, es en la esfera de la política donde termina este consenso, lo que debería considerarse casi inevitable, dada la tendencia permanente dentro de los debates sobre la política cubana hacia la sujetividad ideológica. Una realidad del estudio de Cuba desde 1959 han sido esta tendencia hacia la sujetividad, la dependencia de reportajes parciales y partidiarios, y la falta de acceso a los datos internos sobre la política, la imposibilidad muchas veces de comprobar juicios realizados desde fuera en un ambiente de crítica general a la politica interna de la isla ${ }^{3}$. Así que los estudiosos de Cuba ya conocen bien la escisión profunda que caracteriza la literatura sobre la política cubana, entre una tendencia no crítica (o desde Cuba o desde los grupos de solidaridad) y las críti- cas monocromáticas identificadas normalmente con comentaristas estadounidenses o emigrados. Además, más allá de lo académico, se suele encontrar una dependencia -para nuestras imágenes políticas de Cuba- de la cobertura periodística casual, con frecuencia norteamericana o eurocéntrica, en particular los que, a partir de 1959 , han llegado a la isla en búsca del próximo 'dominó'.

En consecuencia, a diferencia de los debates sobre los cambios económicos y sociales en Cuba, sí se ve un debate fundamental sobre la idea del cambio político. En particular, es común que se cuestione la existencia misma de un proceso de reforma política; los que lo niegan suelen calificar el proceso reciente en la política cubana de una cercenadura ideológica, dominada por un dictador envejecido y un Partido burocrático, oponiéndose resueltamente contra cualquier cambio significativo de un sistema que ya se encuentra bajo presiones internas. La versión más sencilla de esta perspectiva tiende a ser reflejada en las opiniones en los círculos gobernantes en Washington, en el impulso hacia la Ley Helms-Burton, y en los grupos más acérrimos de la emigración ${ }^{4}$. La versión más sútil detecta

* University of Wolverhampton, Reino Unido.

1. En particular en los EE.UU. han aparecido varios estudios recientes sobre la crisis, los cambios y el futuro del sistema cubano, una ola de publicaciones que, en su conjunto, representa la misma variedad de calidad, perspectiva y prognóstico que se encontraba en los primeros años de la Revolución.

2. Aunque aquí se refiere a 1989 como inicio de la crisis, la crisis económica realmente profunda azotó a Cuba desde 1991, con el colapso de la Unión Soviética, produciendo éste una caída económica en Cuba de proporciones desastrosas, en particular entre 1992 y 1995 . Fue, en realidad, en esos años cuando la economía cubana vio sus días más negros. Sin embargo, es también posible considerar que la crisis en la economía empezara a mediados de los años 80, cuando Gorbachev indicó, por primera vez, que la relación hasta entonces cómoda para Cuba, iba a terminar cuando llegara el momento previsto para saldar las cuentas financieras entre los dos países.

3. En realidad, incluso la calificacion de los comentaristas sobre la politica cubana refleja la dificultad, ya que la palabra que suele utilizarse entre tales comentaristas - cubanólogo - es rechazada por los cubanos de la isla, identificándola con los críticos anticastristas en los EE.UU.

4. Esta ley (La Ley de Libertad y Solidaridad Democrática Cubanas) fue propuesta en 1995 por dos políticos republicanos estadounidenses- Jesse Helms y Dan Burton - opuestos al régimen castrista en Cuba; tras el incidente de las avionetas, en febrero de 1996, el presidente Clinton se vio obligado a firmar la ley, aunque sigue en suspenso una parte importante de ella. 
cambios, pero propone que son éstos puramente cosméticos y parciales.

Para poder aproximarnos a este asunto, sin embargo, hace falta ir más allá de los estereotipos, descartándose criterios preconcebidos sobre la base de la ideología o de otras experiencias. Hace falta, en particular, reconocer que si se plantea la pregunta incorrecta se recibe la respuesta incorrecta. Así, en lugar de plantear preguntas sobre un supuesto proceso de reforma (cuestionando su existencia y su eficacia), las que suelen implicar un sistema monolítico, esterotipado y sin cambios, deberíamos enfocarnos a la idea de una estructura política contemporánea que sigue evolucionándose sobre la base de pautas ya establecidas. Según esta perspectiva, resulta más útil plantear las preguntas siguientes: ¿ ¿Sigue el sistema evolucionándose según las líneas anteriores a 1989 o hay ruptura con esa trayectoria? ¿Cuáles son los factores que más afectan la evolución más reciente o esa ruptura?

Es decir que, para mejor comprender lo que pasa actualmente en la Cuba de hoy, hace falta recordar que se habla con más utilidad de un proceso de cambio político desde 1959, en lugar de un sistema de acción y comportamiento político. Simplemente, si se insiste en analizar la política cubana desde la óptica de un sistema, empleando los criterios de 'reforma', 'democratización' o 'sociedad civil', resulta muy fácil engañarse en la búsqueda de elementos que no se identifican fácilmente en esta cultura política tan distinta.

Por consiguiente, el primer paso hacia una comprensión política de Cuba, es la necesidad de descartar los criterios externos, de evitar la tendencia a plantear el caso cubano en contextos inadecuados, que son muchos. El contexto más común en este sentido es la Europa Oriental del período 1945-1989. Aquí es interesante notar que, aunque, tras la tendencia inicial, en los años 1959-1963, de colocar a Cuba en la categoría 'soviética' o 'satélite', se veía en los años 70 una tendencia de aceptar la idea de una revolución distinta y diferente y de analizar la política cubana según criterios distintos, los análisis desde 1989 han sido tentados otra vez hacia las perspectivas iniciales, regresando a expectativas sobre Cuba que están basadas en la experiencia del bloque soviético entre 1989 y 1991. Otro contexto muy común es el de los procesos latinoamericanos de la 'democratización' posmilitar, los que, sin embargo, salieron de realidades muy distintas de la cubana -en sociedades que, antes de la militarización, tuvieron experiencia de sistemas y culturas políticas de competitividad, y que, durante el período de los militares, estaban caracterizados por concepciones de 'seguridad nacional' y de una estrategia militar-tecnocrática de un neoliberalismo que marginaba y empobrecía a muchos de sus propios ciudadanos. Es evidente que, aparte de los debates sobre el significado de la 'democracia', no hay relación entre el régimen cubano antes de 1989 y los varios regímenes militares de Argentina, Chile o el Brasil.

Igualmente, es importante recordar que cualquier cambio político que se distinga no se debe exclusivamente a estímulos externos, ni a la necesidad de adaptarse a la crisis desde 1989 ni a la obedencia a presiones externas hacia la reforma. Sí que han sido críticos estos dos factores, determinando los debates internos y los experimentos recientes, pero no han sido ni los únicos ni los más importantes. Al contrario, hay que reconocer que la dinámica de la política interna y externa de Cuba conduce a una actitud tanto de rechazo como de reconocimiento frente a tales presiones. Hablar de 'condicionalidad' produce un alza de la temperatura nacionalista no sólo dentro de la cúpula cubana sino también en una parte significativa de la población misma. Esta tendencia ha sido evidente en las actitudes generales tanto del público como del gobierno hacia las presiones estadounidenses realizadas en la Ley Torricelli de 1992 y la Ley Helms-Burton de 1996, pero, también en la reacción a la riña reciente entre el gobierno cubano y el recién elegido gobierno español de Aznar.

Así es más importante buscar las fuerzas a favor del cambio dentro de Cuba y no fuera. ¿Cuáles son estas presiones? Primero, hace falta reconocer las que surgen de la serie de tensiones, batallas y debates dentro del liderazgo a lo largo de las cuatro décadas de la Revolución. Aquí es importante ver estas tensiones y diferencias no como escisiones profundas y lineales (ya que no ha habido una división larga de la vanguardia entre dos campos claramente identificables) ${ }^{5}$, sino como un proceso continuo de debates, antagonismos a varios niveles y sobre diferentes asuntos, entrelazándose, contradiciéndose y modificándose. Según esta perspectiva, resulta bastante fácil categorizar las 'divisiones' a partir de 1959 como una batalla larga entre, por ejemplo, fidelistas y el PSP, o entre ortodoxos y radicales, o, alternativamente, entre nacionalistas y comunistas ${ }^{6}$. Sin embargo, hace falta reconocer que no sólo han evolucionado estas diferencias a lo largo del proceso, sino que los protagonistas rara vez se han encontrado claramente dentro de un campo u otro. Además, vale la pena reconocer también que en Cuba la contradicción es muchas veces la esencia del proceso, y resulta más útil ver las supuestas divisiones como debates que inevitablemente van transformándose dentro de un proceso esencialmente reflexivo en una pequeña economía dependiente y así igualmente reflexiva pero dentro de una cultura altamente politizada.

Más allá de esto, además, se ha visto desde los primeros días de la Revolución (y por cierto desde 1962) lo que se puede calificar de un programa de 'reforma' en la percepción continua de la vanguardia revolucionaria de la necesidad de reestructurar, purgar, modernizar el Partido

5. Es importante reconocer la sutileza de estas tensiones, ya que una gran parte de la historiografía de la Revolución enfoca a las diferencias tan destacadas de 1962 y 1968 (cuando hubo cierta enajenación entre los que podríamos llamar los fidelistas y los ex-miembros del (comunista) Partido Socialista Popular, expresada en los dos casos Escalante), y otra vez en el período 1970-1975 cuando los 'vencidos' de esos episodios regresaron a posiciones de influencia en el nuevamente recreado Partido, y en el llamado proceso de rectificación de errores pasados y tendencias negativas a partir de 1986. Lo interesante de la política cubana, sin embargo, ha sido el hecho de que estos períodos de tensión nunca han producidos purgas como pasó en la Unión Sovietica, y, muchas veces, los 'perdedores' de cualquier debate todavía se encontraban en posiciones bastante cómodas, todavía se refería a ellos en la prensa y todavía gozaban de cierto respecto oficial.

6. El PSP (Partido Socialista Popular) era, desde 1944 hasta 1962, el nombre del Partido Comunista en Cuba. 
(en cualquier forma que se haya presentado este, o sea ORI, el PURS o el PCC $)^{7}$. Naturalmente, esta tendencia ha reflejado a menudo los debates realizados en la cúpula, una situación en la cual el argumento prevaleciente se trataba siempre de consolidar su ascendencia por reconfigurar el partido según sus propios criterios. A veces, además, tal tendencia hacia la transformación del partido ha respondido a un momento interno, a la lógica de la transformación, o a presiones de base (contra la incapacidad del Partido de reflejar los intereses del pueblo), y a veces a la necesidad de eliminar la ineficiencia. La realidad es que, en los sectores medianos del Partido, se ha visto siempre un proceso de cambio de personal, cualquiera que fuera el carácter del partido. Efectivamente, las presiones ejercidas también desde fuera del Partido -de los Comités de Defensa de la Revolución, de la Central de Trabajadores Cubanos o de las Fuerzas Armadas Revolucionarias- han asegurado un proceso permanente de cambio de la militancia ${ }^{8}$. Igualmente, en el sistema representativo, los Organos de Poder Popular (OPP), el nivel municipal ha visto también muchas veces un proceso destacado de cambio de personal, en particular a través del sistema de Rendición de cuentas?.

Estas dos tendencias - el interno dentro del Partido y el estructural en el sistema electoral - han tenido dos efectos. Primero, han asegurado la no formación de bloques votantes en las asamblea elegidas y también la falta de profesionalización de los representantes. El segundo efecto ha sido el fortalecimiento de la autoridad y la continuidad de los que han quedado, sobreviviendo a los cambios, lo que ha significado que los militantes del Partido, por ausentes que sean entre las filas de las asambleas municipales, han tendido a dominar las provinciales y la nacional. En realidad, antes de 1993, cuando se decretó una reforma del sistema de representación a nivel nacional, eran fundamentales a las contradicciones del sistema político las tensiones entre los niveles inferiores de los OPP -elegidos directamente, y, así más accesibles, responsables y transformables) - y el nivel nacional (elegido indirectamente, más permanente y menos responsables).

Finalmente, en el vórtice del proceso de rectificación desde 1986 , se produjo una serie de nuevas presiones ${ }^{10}$. Por ejemplo, era evidente que al proceso revolucionario, y en particular al Partido y a la vanguardia, les hacía falta adaptarse a los mismos cambios sociales profundos que la Revolución había realizado a lo largo de tres décadas, a responder a una juventud cada vez más enajenada y menos interesada por el lenguaje de sus padres, a la movilidad de la población afrocubana, a la frustración política de una población femenina que todavía se sentía desigualmente representada. Tales presiones, predatando la crisis, incrementaron y se agudizaron a partir del año 1989, a medida que la crisis y los cambios económicos rompieron las expectativas, minaron los principios establecidos y aceleraron el proceso de redefinición. Con el nuevo aislamiento, ya que la Revolución se encontraba privada de la antigua protección socialista y asediada por un bloqueo cada vez más fuerte, desaparecieron los modelos conocidos.
Ya se produjo una situación completamente nueva, acarreándose una gama de nuevos imperativos. Primero, se vio el deseo predecible del liderazgo de ampliar el frente político en Cuba -aunque, fuera de la isla, muchos comentaristas habían esperado todo lo contrario, prediciendo, sobre la base de la imagen de la rectificación (con sus implicaciones de purga), un estrechamiento de las perspectivas, de la vanguardia y de las definiciones. Fue esta búsqueda de la unidad ante la crisis, ante el asedio reimpuesto y ante el aislamiento renovado la que motivó el lanzamiento del Periodo Especial (con sus recuerdos del ambiente general de los años 60) ${ }^{11}$. No era pura nostalgia por una edad de oro, sino un reflejo de un motivo pragmático -el de persuadirles a suficientes cubanos -todos presionados, agobiados y desmoralizados-de que reconocieran la necesidad de defender colectivamente los beneficios ya realizados pero ahora en peligro de desaparecer.

El segundo imperativo era el impulso de parte del liderazgo de reafirmar su propia legitimidad, así como la del Partido y de la Revolución misma, frente a la perestroika, a la nueva relación con Moscú y al caso Ochoa en

7. En 1961, las tres fuerzas principales de la rebelión (el Movimiento 26 de Julio de Fidel Castro, el PSP y el pequeño Directorio Revolucionario Estudiantil), se unificaron en las Organizaciones Revolucionarias Integradas (ORI), organismo cuya creación, bajo la dirección de Aníbal Escalante del PSP, provocó el primer caso Escalante. Luego en 1962, tras este episodio, las ORI fueron sustituidas por el Partido Unificado de la Revolución Socialista, el que fue también sustituido en 1965 por el recreado Partido Comunista Cubano, organismo que, a pesar de su nombre, era constituido más por un Comité Central dominado por la vanguardia de la lucha guerrillera de 1956-8 que por una estructura masiva de base. Por ejemplo, el primer congreso nacional del PCC se celebró en 1975.

8. Los CDR fueron creados por el gobierno en 1960, como otra arma de defensa contra la invasión que, según la inteligencia, estaba en preparación en Florida y Centroamércia. La intención era la creación de comités en cada cuadra de las ciudades y cada aldea, a los cuales pertenecerían todos los habitantes leales de la cuadra o aldea, con la responsabilidad de identificar los que simpatizarían con la invasión, una posible 'Quinta Columna'. Cuando esa invasión se realizó, en Playa Girón, en abril de 1961, los CDR sí ejercieron un papel de vigilancia muy fundamental a la victoria de las fuerzas revolucionarias, acusando y deteniendo miles de los opuestos a la Revolución. A partir de 1961, sin embargo, los CDR se convirtieron en los organismos mas extensos y más eficaces de base del proceso revolucionario, organizando campañas de toda índole y logrando asegurar la participación masiva. En 1976, sus funciones fueron en parte sustituidas por el sistema de Poder Popular.

9. La rendición es el mecanismo por el cual, cada seis meses, los delegados municipales deben acudir a una asamblea de sus electores para defenderse ante su comportamiento como representante; si un $50 \%$ del electorado vota en contra de tal delegado, lo sustituyen por otro. En los años 80 , se vio un cambio significativo de tales representantes.

10. El Cuarto Congreso del Partido, en 1986, lanzó una campaña de rectificación, la que se veía fuera de Cuba como una resistencia a la influencia de la perestroika y de la glasnost, pero que fue en efecto la continuación de tendencias vistas en los años 60 y 70 y una reacción contra el crecimiento dentro del Partido del privilegio e incluso de la corrupción. La existencia de cierta resistencia a esta campaña era evidente en el hecho de que, tras la primera sesión del Congreso en febrero era necesario reconvocarlo en diciembre, oficialmente para permitir un período necesario de consulta nacional.

11. El 'Período Especial en Tiempos de Paz' es la estrategia concebida para responder colectivamente a la crisis doble - de la economía y del aislamiento político - y consiste más o menos en el desarrollo de una 'economía de guerra', reduciendo las expectativas, las demandas y las pérdidas para motivar un sentimiento de unidad popular frente a la crisis. Incluso se hablaba oficialmente, mientras la economía esperaba lo peor de la caída (entre 1989 y 1992), de la posibilidad de una 'Opción Zero', una retirada económica total hasta el nivel básico. 
$1989^{12}$. En parte, el proceso de rectificación había abordado este tema, pero era enorme el daño posible a la legitimidad que pudieran causar las crisis sucesivas y destabilizadoras, seguidas por el colapso del CAME (el Comecón) y de la Unión Soviética.

Por lo tanto, un tercer imperativo era el deseo de la vanguardia recién restaurada de asegurar que los que abogaban por la glasnost o la perestroika no lograsen corromper esta unidad y esta legitimidad frágiles ${ }^{13}$. Tras 1991, les perseguía el espectro de Gorbachev -el de un proceso de reforma que respondiera a presiones externas sólo para provocar una implosión política del sistema mismo- a pesar de comprender bien que las condiciones cubanas no eran las mismas de Polonia, de Rumania ni de la Unión Soviética. Para éstos, y de éstos, el mensaje era sencillo -sería una tontería peligrosa atreverse hacia una reforma parecida. Era un mensaje que se repetía constantemente.

Finalmente, se veía una necesidad sumamente pragmática, la de asegurar que se realizaran dos objetivos casi contradictorios: la promesa de una evolución política para asegurar la existencia de tiempo suficiente para que las reformas económicas se verificaran y produjeran efectos positivos (dándoles a los cubanos comida y trabajo), y la defensa del frente, políticamente, hasta que tales reformas económicas implicaran la necesidad inevitable del ajuste.

Es éste, por lo tanto, el contexto amplio y contradictorio dentro del cual, desde 1985, se manifiesta la necesidad continua del proceso político cubano de evolución, e incluso de reinventarse. Así es evidente que ver a Cuba según las perspectivas de la 'democratización' o de la 'reforma' corre el riesgo de malentenderla, un riesgo que se exacerba cuando se recuerda la existencia ya evidente de cuatro realidades fundamentales que han contribuido a determinar la naturaleza del cambio polítco en Cuba.

La primera de estas realidades es el punto de partida para cualquier análisis del sistema político, es decir el reconocimiento de que el sistema cubano no es hoy, y nunca ha sido, una estructura monolítica, unidimensional, sino al contrario una red compleja de mecanismos paralelos de comunicación y representación, todos entrelazándose, contrapesándose y reforzándose, y una red, igualmente compleja, de niveles de poder y participación y niveles de actividad política. Un ejemplo claro de esta realidad fue la creación de los OPP en 1976, que no eliminaba ni los CDR anteriores ni tampoco las tensiones entre éstos y el Partido. Efectivamente, considerando la complejidad del sistema político, resulta más útil considerarlo no según criterios de estado y sociedad civil sino en términos de 'espacios políti$\cos ^{14}$ y niveles, o 'circuitos', de participación y poder ${ }^{15}$.

La segunda realidad es una que frecuentemente se olvida, por lo evidente que es. Es esta la ausencia de un proyecto coherente y significativo de oposición, organizado nacional o aún regionalmente. Se debe esto, seguramente, a los efectos de décadas de una emigración masiva, la que ha logrado eliminar la posibilidad de la organización de tal proyecto. Un factor fundamental que tienen que considerar cualesquiera que piensen organizar una campaña de oposición en Cuba es el hecho de que, con una frecuencia notable, cuando se nota el crecimiento de una disidencia real o potencial le suele seguir un momento de lo que podemos llamar una 'sangría' aprobada, según la cual se permite amigrar a los que disienten, debilitando así un posible frente opositor interno. Por consiguiente, cualquiera que sea la escala de la disidencia, la oposición o la discrepancia, la realidad es que la válvula de seguridad ofrecida por la emigración les ha privado a los disidentes posibles del impulso y de los mecanismos para la creación de una fuerza significativa de oposición ${ }^{16}$.

La ausencia de tal proyecto opositor tiene tres efectos principales. En primer lugar, tanto la política oficial como la literatura sobre Cuba están caracterizadas por una incertidumbre continua acerca del nivel de oposición real y eficaz - a diferencia de las quejas perpetuas que se suelen oír en todas partes de la isla en cuanto a medidas específicas, al mismo Fidel Castro, a carencias y así en adelante. En segundo lugar, esta ausencia provoca otra, la de una fuente necesaria de información política para el mismo liderazgo, que le permitiría leer y saber adaptarse o responder a las demandas populares. La necesidad de tal canal informati-

12. En julio de 1989, Arnaldo Ochoa, uno de los militares más prestigiosos de Cuba, y uno de los pocos Héroes de la Revolución, y también el encargado de la operación permanente de romper el bloqueo norteamericano, fue detenido, procesado y sentenciado a la pena de muerte por la corrupción, específicamente por involucrarse en el narcotráfico colombiano y por haber abusado de su posición privilegiada. Muchos comentaristas vieron en este episodio una maniobra en contra de las FAR, para disuadir a cualesquiera militares dispuestos a quejarse de su situación en una economía en crisis tras haber regresado, como héroes, de las campañas angoleñas. Otros lo consideraron la eliminación por Castro de un rival potencial. Aunque es posible que también sirviera para avisar a los militares que no lanzasen un reto al sistema, parece probable que las acusaciones fueran correctas y que, atacando a un líder prestigioso de esta forma, la vanguardia iba difundiendo un mensaje a todos los quejumbrosos, ambiciosos o descontentos de la estructura política, de que el sistema no iba a tolerar ni la corrupción, ni el individualismo, ni simpatías por una reforma al estilo Gorbachev. Efectivamente, en el mismo episodio cayeron del poder muchos del Ministerio del Interior y de las FAR, tal vez las dos instituciones más identificadas con Moscú y, seguramente, las más poderosas aparte del Partido (ya en vías de 'purificarse' por la rectificación).

13. En 1975, con el Primer Congreso del Partido, el nuevo sistema electoral (OPP), que evidentemente tenía como modelo el soviético, y con la participación cubana en el CAME, los cuadros de la vanguardia que había sido marginados entre 1962 y 1970, se encontraban otra vez en posiciones de influencia, tanto en el Partido como en la dirección económica. Sin embargo, a partir de los años finales de la década, era posible discernir un regreso parcial de elementos, actitudes y políticas más asociados con los fidelistas, un proceso que se aceleraba despues de 1984 y que se cumplía con el Congreso de 1986.

14. Tal vez el mejor estudio reciente de la complejidad del sistema cubano es 'Back from the Future. Cuba under Castro', de Susan Eckstein (Princeton University Press, 1994).

15. Aquí se refiere al criterio que viene elaborando este autor según el cual se aplica al sistema político cubano el modelo de los 'dos circuitos' que elaboró Milton Santos en su estudio de la informalidad y la urbanización en Latinoamérica, 'The Shared Space' (1979), el que consiste en la coexistencia de dos circuitos de actividad económica que se intersecan, o sea el 'formal' y el 'informal'.

16. Varios estudios han calculado el nivel de los que discrepan de la Revolución entre un $15 \%$ y una tercera parte de la población, dependiendo esto del momento dado. Por ejemplo, era posible calcular, en las elecciones de OPP en febrero de 1993 que un 19\% habían logrado votar en contra de la oficialidad, una cifra confirmada por los criterios del disidente más destacado en aquel momento, Elizardo Sánchez. Es casi cierto que el daño causado por la crisis y las reformas desde aquel año han provocado una alza de la cifra, aunque es también probable que la cifra haya sido más o menos constante a partir de las grandes emigraciones de la primera década. 
vo es evidente a todos los que conozcan la realidad cubana, que está a veces caracterizada por lo que podemos llamar una 'inercia informativa', según la cual los órganos oficiales pueden preferir repetir los 'mensajes' que perciban son los que busca la vanguardia, prefiriendo quedarse dentro de los parámetros institucionalizados de la opinión ${ }^{17}$. En tercer lugar, ha asegurado la ausencia de una presión bien organizada por el cambio, de parte de un movimiento (o una entidad) dispuesto a cuestionar fundamentalmente a la estructura política.

En consecuencia, cualquier insatisfacción popular suele expresarse visible pero incoherentemente a través de la protesta callejera esporádica o de la protesta laboral -siendo las dos ocurrencias muy raras- $\mathrm{o}$ a través del austentismo laboral. Al contrario, significa que toda presión por el cambio, el ajuste, la reforma o la evolución debe provenir desde dentro de un sistema que está tan caracterizado por redes y canales complejos, por obstáculos institucionales y por tabúes dentro de los parámetros definidos o del contexto geopolítico como para asegurar que tales presiones sean difíciles de reconocer o de interpretar ${ }^{18}$.

La tercera realidad subyacente es, y debe ser, el factor constante del nacionalismo. Es constante no en su naturaleza inalterable (ya que se han manifestado muchas modificaciones y muchas formas diferentes), sino en su importancia. En el período 1959-1963 fue el nacionalismo el elemento fundamental para comprender la radicalización inicial, siguiendo como el cemento permanente a lo largo de los períodos de una transformación posiblemente destabilizadora. Incluso hoy sigue siendo un factor capaz de sorprender, de distorsionar las expectativas y de complicar las reaccciones populares, y es muy posible que sea todavía un factor que ha logrado retrasar la emergencia de divisiones políticas que, en tiempos de crisis y trauma, se pudieran esperar.

La cuarta, y final, realidad fundamental que ha determinado la evolución política son los efectos de la participación, de la serie extensa, amplia y eficaz de medios, canales, órganos y foros de participación, de comunicación y de movilización desde los primeros momentos de la Revolución hasta hoy mismo. Es casi seguro que esta presión constante por participar, estas formas de expresarse, aunque limitadas, y esta cultura de campañas y de movilización permanente han logrado apaciguar el descontento, desviándolo, desactivándolo e incluso disfrazándolo, o tal vez permitiendo la participación genuinamente popular, así reduciendo la demanda posible por una 'democratización' al estilo latinoamericano o como se vio en la Europa Oriental. Pueden ser a veces poco eficientes o eficaces las medidas de participación pero sí que existen y ha contribuido esto a la falta de una reivindicación generalizada y urgente de más 'democracia'.

El significado de todo esto es que es probable que todos los cambios que logremos percibir sean realmente mucho más significativos de lo que parezcan ser al principio y que pueda haber un proceso de reforma que no se parezca pero que lo es. Es decir que, aunque puede que los cambios aparentemente ligeros que se distingan no hayan tenido un impacto muy visible, es posible afirmar que, en realidad, lo que viene ocurriendo es una transformación profunda hacia una estructura política algo diferente de la conocida.

Entonces, ¿cuáles son los elementos de cambio -posiblemente significativos- que se han visto en los años recientes, específicamente desde 1992 ?

El primer cambio -primero por ser el más publicado con un efecto inmediato- fue la reforma muy necesaria de la Asamblea Nacional en 1992, permitiendo por primera vez la elección directa de los delegados. Como parte de esta reforma también se dictaba la creación simultánea de comisiones permanentes de la Asamblea para efectuar el trabajo de ésta, mecanismos tal vez no tan eficaces como una Asamblea permanente (ya que sigue la pauta de una convocación dos veces al año por unas semanas cada vez), pero constituyendo la reforma un paso importante hacia la permanencia necesaria. Aunque fuera de Cuba esta reforma fue a veces muy criticada, descartada como un cambio cosmético, en realidad tuvo, y sigue teniendo, un efecto importante en muchos sentidos. Por ejemplo, es evidente que ha abierto el debate público en cuanto al valor y significado de la representación dentro de una estructura hasta entonces orientada hacia dos formas de democracia -la 'directa' que caracterizaba los 60 (las asambleas públicas en la Plaza de la Revolución, las movilizaciones masivas, los viajes imprevistos de los líderes a traves del país)- a la que le faltaban la coordinación nacional y los canales estructurales de comunicación, y la institucional o selectiva que había caracterizado el período que seguía el regreso al poder de los elementos más 'ortodoxos', más prosoviéticos, donde antes faltaba la coordinación efectiva a nivel nacional. Igualmente, ha creado nuevas responsabilidades y un nuevo poder para los delegados que ya cuentan con una base a la cual son más responsables que antes y más conscientes del propósito de la Asamblea.

El efecto más inmediato se vió en febrero de 1993 , cuando una votación otra vez tan extensa como en el pasado indicaba cierto rescate de un sistema parlamentario que había perdido algo de su legitimidad, por ser poco sensible y poco eficaz. A diferencia de las elecciones municipales unos dos meses antes, los comicios de febrero, tras una campaña fuerte de auto-crítica y de persuasión, y tras unos esfuerzos extensos por parte de los CDR, manifestaban una votación de un $98,8 \%$, un $81 \%$ de los cuales aceptaron la lista oficial de candidatos ${ }^{19}$. Aunque es posible que estas cifras sean algo exageradas y manipuladas, parecía

17. No es siempre este el caso, sin embargo, ya que los episodios de consulta nacional, frecuentemente en momentos de crisis o tensión por ejemplo, después del fracaso de la gran zafra de los 10 millones de 1970, o antes del Cuarto Congreso (1991) - y el proceso actual de reexaminación nacional han visto muchas críticas, sugerencias y presiones, las que han logrado cambiar aspectos de la política oficial.

18. Unos ejemplos de tales protestas se vieron a finales de los 60 , cuando incrementaba notablemente el ausentismo laboral, seguramente en parte protestando contra las presiones y la austeridad, y también en el verano de 1994 cuando la turbulencia callejera en La Habana Vieja formó una parte del episodio de los balseros.

19. En diciembre de 1992, en plena crisis económica y tal vez el peor momento de los nuevos días negros, las elecciones municipales registraron una tasa peligrosa de abstencionismo (un 30\%) y de rechazo (un 50\% de los delegados necesitaban una segunda votación para confirmarse). 
que la reforma había tenido buena acogida de un electorado dispuesto a concederle al sistema otra oportunidad. Es cierto que esta imagen fue reforzada no sólo por las aseveraciones de unos críticos cubanos, quienes aceptaban los resultados, sino también por los resultados de las próximas elecciones municipales, en julio de 1995, las que vieron una abstención de sólo un 5\% en La Habana (siempre la región más crítica y más discrepante), junto con un total de un $11 \%$ de votos blancos o manchados. En comparación con el desastre electoral de 1992, estos resultados parecían indicar cierta buena voluntad de parte de los votantes de confiarse en un sistema que ahora gozaba de más pertinencia, más legitimidad y más vigor, probablemente como resultado de un proceso cuidadoso hacia la reinvigorización del sistema local desde 1993, respondiendo no a demandas 'democráticas' sino a su eficacia en aspectos económicos, tratando de convertir los OPP en fuentes de asesoramiento y mecanismos eficaces para romper los obstáculos ineficientes. Realmente, entre 1990 y 1994, la credibilidad de los OPP a nivel local había bajado notablemente; ahora se resucitaba.

Otra reforma de esta índole se vio en 1993 con la creación de los Consejos Populares. Para los que sólo tuvieran una idea imprecisa de la Revolución Cubana, era lógico suponer que estos órganos eran un mecanismo típico de la imagen y la mitología de una revolución participativa; sin embargo, en la realidad eran mucho más una parte de la institucionalización burocrática y gerencial, pero, paradójicamente, más necesarios y más populares por serlo. Eran los Consejos asambleas administrativas, una solución práctica a las dificultades locales y regionales de la distribución de bienes y servicios, reuniendo a los delegados de los OPP y los CDR con los administradores de las empresas locales y los cuadros dirigentes del Partido, todo para producir un instrumento para el funcionamiento del sistema económico. Considerando su propósito muy inmediato, se convirtieron rápidamente en mecanismos muy eficaces y con cierta legitimdad popular, generando a su vez la reinvigoración ya mencionada de los OPP.

Pero ¿cómo podrían ser políticamente significativos? En primer lugar, su significado se encontraba en su concepción, ya que su creación fue realmente una admisión pública de la ineficacia e ineficiencia de los sistemas existentes de representación, y respondieron en parte a las demandas expresadas a través de los OPP y los CDR. Dada la autocrítica implícita en el hecho de la reforma de la Asamblea Nacional, dio impulso este cambio a un debate bastante abierto acerca de la naturaleza, la eficacia y la función de la representación, un debate que incluso ha generado un interés por los sistemas de representación local en otros países.

El otro significado de los Consejos era a más largo plazo, en su capacidad de ofrecer un modelo posible de cooperación práctica y aún corporativa, una lección que tiene significado para los que consideran una ampliacion del sistema político por motivos pragmáticos.

El tercer cambio formal de importancia ha sido la creación de los Parlamentos Obreros, una reforma que generalmente no ha sido muy creída fuera de Cuba y vista como otra medida cosmética, lo que es una reacción natural dada la historia difícil de tensiones y contradicciones entre gobiernos socialistas y órganos de representación obrera tanto en la Europa Oriental como en la misma Cuba. Este escepticismo, además, ha sido reforzado por la utilización evidente de estas asambleas laborales como otro instrumento para preparar a los obreros cubanos para duras medidas económicas, por fomentar la discusión previa de tales medidas e incluso persuadiéndoles a los mismos obreros que las propongan ellos. Sin embargo, son los Parlamentos tal vez la punta de un iceberg más significativo de lo se supone, ya que se desarrollan ya más allá de lo que fue tal vez previsto en sus propósitos y su utilización originales.

Por ejemplo, aunque es claro que tenían la meta inicial de servir como válvulas de escape, para permitir la discusión de temas difíciles entre un sector de la población que ha estado tanto en pleno centro del colapso económico como en la primera línea del esfuerzo productivo, los órganos han logrado, muchas veces, convertirse en foros de consulta genuina, en parte para permitirle a la vanguardia que juzgue la acogida de ciertas medidas o, alternativamente, la resistencia probable a éstas. No obstante esta función, lo que les ha pasado ha sido un movimiento paralelo al crecimiento del signficado del periódico Trabajadores durante la crisis, o sea que han sido, en parte, apropiados por los mismos obreros (a través de la CTC), para permitirles la posibilidad de quejarse, de apuntar los efectos adversos sociales de ciertas medidas. Así es posible ver estos Parlamentos o como foros de consulta corporatista o como foros de debate real y de críticas no expresadas en otro lugar, así como de una conciencia social del proceso de reforma económica. Por lo tanto, todos estos órganos obreros -los Parlamentos, el periódico y la CTC misma han servido cada vez más como pretexto, testimonio y apoyo para los argumentos de los que se oponen a las reformas económicas y también como un freno a los que abogan o por la liberalización desenfrenada o por la resistencia total a la reforma en general.

Más allá de estas funciones, los Parlamentos también desempeñan otro papel, característico de muchos cambios recientes, el de la experimentación, ya que el proceso largo de la Revolución tiene una historia bien clara de una tendencia hacia la experimentación, proponiendo proyectos experimentales en lugares definidos de la isla antes de extenderlos a toda la nación ${ }^{20}$. Así es muy posible que los Parlamentos, si tienen éxito en cuanto a su acogida, su legitimidad popular y su eficacia, puedan servir de modelo para otros cambios en la estructura política.

Así, examinando estas tres reformas desde cerca, reformas frecuente y fácilmente descartadas como poco pertinentes y superficiales, se puede ver en ellas elementos indicativos de una voluntad del sistema para la experi-

20. Dos ejemplos destacados de esta tendencia se vieron en 1974 con un estudio experimental de los OPP en Matanzas, dos años antes de establecer el sistema nacional, y, más tarde, con la experimentación con los Consejos Populares en barrios selectos de La Habana. 
mentación, la expansión, la invención y el reconocimiento de la ineficacia de los sistemas existentes.

Sin embargo, por debajo de estas reformas, también es posible identificar una serie de procesos de ajuste que ya están bien establecidos y datan del período anterior a la crisis. El proceso más visible ha sido la emergencia de un liderazgo más joven y, a la vez, más colectivo. La 'rejuvenización' ha sido, según muchos comentaristas y no pocos cubanos, una transformación ya atrasada, dado el sistema repleto de peligros de la 'gerontocracia', dominado o por la misma generación de la rebelión guerrillera (la 'vieja vanguardia') o por elementos del Partido que en ciertos momentos miraba hacia Moscú para sus criterios. Así ha sido evidente la posibilidad creciente de conflictos entre la retórica de una Revolución que exaltaba la juventud pero que seguía dominada por una vanguardia entrada ya en años pero sin dárse cuento de ello, entre, por un lado, las expectativas materiales de la nueva generación, educada para buscar carreras prometedoras y, por otro lado, tanto la realidad de una economía todavía agudamente tercermundista como la sobrevivencia del simbolismo y del lenguaje de otra época no conocida por ella. Así, es muy significativo el reconocimiento gradual de la necesidad de responder a esta generación potencialmente enajenada por elevar a un perfil más destacado y por darles más responsabilidad para aspectos clave de la estructura y del gobierno a unos políticos más jóvenes -Roberto Robaina, Carlos Lage, Abel Prieto, Vicky Velázquez, e incluso Ricardo Alarcón ${ }^{21}$. Es cierto que la subida de esta generación, confirmada por los cambios más recientes del Quinto Congreso del Partido, ha sido uno de los cambios sistémicos de más envergadura.

En cuanto a la dirección colectiva, siempre ha contradicho la realidad a la imagen convencional de un sistema dominado por una sola persona, una realidad que respondía tanto a las simplificaciones externas como a las internas. Es decir que el enfoque perpetuo a Fidel Castro, tanto por los extranjeros como por los mismos cubanos, ha sido tal vez predecible y aún necesario, pero ha simplificado excesivamente una realidad más compleja, ya que, en vez de una situación en la que Castro les ha impuesto a los demás sus decretos, se puede ver que, desde los primeros momentos del proceso, las decisiones siempre han sido debatidas dentro de un determinado grupo dirigente, a veces votando la mayoría en contra de Castro o logrando persuadirle.

Otro proceso de evolución ha sido evidente en la reorientación de la Revolución hacia una perspectiva más claramente nacionalista, en la retórica pública, en la Constitución $^{22}$, en las relaciones con la población emigra$\mathrm{da}^{23}$. En parte se debe este enfoque a las exigencias del momento, haciendo de la necesidad una virtud (respondiendo al vacío producido por el colapso del modelo europeo del socialismo y de la protección ideológica de la comunidad socialista). También se debe evidentemente a la dinámica interna del proceso revolucionario, ya que en parte la historia del proceso revolucionario puede presentarse como la tensión o el debate entre un argumento nacionalista (definiéndose diferentemente según la hora y según el enemigo) y un argumento más orientado hacia modelos externos y, según esta pauta, la desaparición a partir de 1985 del segundo argumento, creó la oportunidad para relegitimar el hilo largo e histórico del nacionalismo. Ya en las actitudes formales de la cúpula ante la crisis inmediata del 1989, en el recurso a los mecanismos y la ética de los 60, se veía cierta identificación con este hilo. Según este criterio, la movilización ante al aislamiento, el sitio y la crisis no era sólo un retorno nostálgico a la 'economía moral' de los 60 (aunque era verdad que la figura de Che Guevara sí que se volvió a ser enfoque de la atención académica), sino también un impulso hacia la unidad nacional $^{24}$.

Esta tendencia se vincula con otra evolución fundamental -la disponibilidad de la vanguardia de ampliar el debate. Es este otro aspecto de una realidad mucho más compleja de lo se suele presentarse en la literatura, ya que aquí nos encontramos frente a una Cuba diferente a la de la imagen monolítica. En vez de una estructura gobernada por el decreto, lo que se ve es un sistema cuya dinámica ha oscilado entre, por un lado, unos períodos de una mentalidad intensa de asedio, cuando se ha encerrado el debate o se ha limitado dentro de los parámetros conocidos y los grupos favorecidos, y, por otro lado, unos momentos, o aún períodos bastante largos, de debate abierto y muchas veces aprobado oficialmente. Este debate se ha visto tanto dentro del Partido (lo que fue evidente en los Congresos de 1986 y 1991), a nivel de la vanguardia así como en la base, y, más típicamente, en círculos definidos académicos, en particular los muchos Centros. Por lo tanto, cuando Raúl Castro dio su discurso en vísperas del incidente de las avionetas, de los Hermanos al Rescate, en febrero de 1996, el que provocó una alarma acerca de una línea dura posible, y cuando se vio cierta presión sobre algunos contactos académicos con el exterior en 1997, no fueron estos indicios

21. Roberto Robaina, ex-líder popular de la Federación Estudiantil Universitario (FEU) y de la Unión de Jóvenes Comunistas (UJC) es ahora Ministro de Asuntos Exteriores; Carlos Lage, también cuarentón, es el responsable de la estrategia de reformas económicas; Abel Prieto, poeta, ex-presidente de la Unión de Escritores y Artistas Cubanos (UNEAC) es Ministro de Cultura; Vicky Velázquez, también, como Robaina, ex-presidente de la FEU, es evidentemente una cubana de futuro importante en el Comite Central del PCC; Ricardo Alarcón, exEmbajador de Cuba en la Organización de Naciones Unidas, es ahora presidente de la Asamblea Nacional y tambien el líder responsable más que nadie para las discusiones con Washington y con los emigrados.

22. En 1991 la Constitucion fue cambiada para enfatizar más, en sus preámbulos, la tradición nacional cubana, junto con la tradición socialista de la cual la Revolución era heredera.

23. En 1994, se celebró la primera conferencia de una serie de eventos anuales con el título, 'Nación y Emigración', dedicados a un reexamen de la comunidad emigrada y al diálogo positivo con elementos de esa comunidad.

24. La 'economía moral' se refiere a la estrategia económica cubana adoptada a partir de 1966, siguiendo el llamado 'Gran Debate' público entre los que abogaban por una estrategia bastante ortodoxa (según criterios soviéticos y socialdemocráticos) y los, incluyendo a Guevara, que creían en una estrategia más industrializada, más centralizada y basada más en la conciencia revolucionaria. La 'economía moral', aunque parecía seguir los argumentos de Guevara hasta cierto punto, era una reacción más a la crisis económica de aquel año y a criterios políticos del liderazgo que a la victoria supuesta de los 'radicales' del Debate. Durante el proceso de rectificación a partir de 1986 , se vio un incremento marcado de estudios sobre Guevara, y, cosa nueva, sobre sus ideas y no sólo su figura y su vida. 
de una línea dura, sino, más bien, una señal de tensiones internas, $\mathrm{y}$ de reacciones al asedio renovado $\mathrm{y}$, a fin de cuentas, una oscilación más del péndulo.

Un proceso parecido de ampliación también se ve en otro contexto: en la tendencia creciente de darles vida y significado a las muchas organizaciones del estado (aparte del Partido) o las organizaciones paraestatales, por ejemplo la CTC, los CDR, y las FAR. Si se sigue considerando el sistema dentro de la perspectiva de una estructura monolíti$\mathrm{ca}, \mathrm{tal}$ proceso puede verse como otra medida puramente cosmética. Sin embargo, tal interpretación se olvida del papel a veces muy importante que han desempeñado estas organizaciones dentro del sistema complejo y fluido de los 'circuitos duales'. Aunque es verdad que frecuentemente pueden ser utilizadas por la vanguardia como válvulas de escape o mecanismos de defracción, muchas veces han logrado descubrir una vida propia, respondiendo -a veces mejor que el Partido (que tiende a ser más programado y más selectivo) - a las reivindicaciones y los intereses de la base. Es verdad esto, en particular, cuando se trata de los CDR y la CTC, ya que, contando con un contacto popular más frecuente, más amplio y menos filtrador por la falta de selectividad, han podido servir como canales eficaces de la comunicación para las preocupaciones, el descontento y las presiones populares.

Históricamente, las organizaciones más importantes son los CDR, aunque es verdad que, actualmente, han perdido algo de esa importancia, en comparación con su apogeo en la década de los 60, cuando sirvieron como mecanismo principal y a veces como el foro único, de debate político, de movilización popular, de socialización política y de participación masiva. Tras un período cuando perdieron también su legitimidad y su utilidad ante los ojos de muchos cubanos, hoy en día, revivándose, funcionan más como mecanismos visibles en la lucha oficial y popular contra el delito creciente en la época de crisis y reforma. No obstante, con su historia, su envergadura y su nivel de participación, tienen todavía la capacidad de movilizar a más de siete millones de cubanos regularmente, así que sería un error pasarlos por alto.

La Central de Trabajadores Cubanos (CTC), el organismo del Estado de más significado (después del Partido) y más importancia simbólica, como representante de los actores clave de la mitología política y la realidad productiva, ha visto un incremento visible y marcado de su importancia en los años recientes. Evidentemente, todas las advertencias pertinentes cuando se habla de la importancia de los Parlamentos Obreros aquí tambien se aplican, pero la diferencia con la CTC es que es una organización nacional, con su propia burocracia, sus propios congresos y su propia dináminca interna, así asegurando que sus líderes desempeñen un papel significativos en los debates de la vanguardia, del Comité Central del Partido y de la Asamblea Nacional. Simplemente, cuando los dirigentes sindicales expresan en uno de estos foros las quejas de sus miembros ante algún problema o ante alguna que otra medida, los demás líderes se ven obligados a prestarles atención. Además, el papel que se le ha concedido desde 1989 a la CTC y a Trabajadores significa que, si deben seguir con cierta legitimidad como defensores de los intereses de la clase obrera cubana, el sistema no tiene más remedio que escucharlos visiblemente.

Naturalmente, como estructura militar, las FAR son mucho menos capaces de reflejar las preocupaciones de base. No obstante, dentro de la ecuación política cubana, es importante verlas como un grupo de presión poderoso, representando a ese sector de la población que, desde 1961 , se ha visto expuesto en los momentos mejores y peores de la Revolución - los oficiales militares. Gozando de un prestigio inalcanzable (gracias a la lucha continua, a Girón, a la Lucha contra Bandidos ${ }^{25}$, a la mentalidad de asedio, a los años del 'internacionalismo' ${ }^{26} \mathrm{y}$, más gloriosamente, a la guerra en Angola) ${ }^{27}$, estos militares también han sido vistos siempre como organizadores de una mano de obra económica masiva y de gran importancia. En 1989, experimentaron tal vez su momento más negro con el caso Ochoa, pero, desde 1992, su prestigio ha crecido de nuevo sobre la base de sus éxitos económicos. Ya, en los 80, era evidente que las FAR tenían una actitud más abierta hacia la reforma económica, en particular ampliando la vista de unas reformas de la administración; ahora, con las reformas de empresas y de la tenencia de tierra, es evidente que las FAR cuentan con los aspectos más exitosos de la nueva economía, en particular, en la agricultura. Además, como organizadores del llamado Ejército Juvenil de Trabajo, siguen teniendo un aporte fundamental a la producción de bajo coste. Tampoco se debe olvidarse del papel, un poco más evidente en los años recientes, de la Asociación de Combatientes de la Revolución Cubana, organización cuyo perfil indica tal vez cierta resistencia a unos cambios de parte de los que se han visto progresivamente marginados por el proceso de reforma así indicando además tal vez cierta determinación entre ellos de conservar lo más posible del proceso por el cual lucharon en el pasado.

25. Entre 1961 y 1966 , había una campaña casi permanente de las FAR y las milicias contra las varias y repetidas rebeliones, conjuras y guerrillas que organizaron los emigrados, infiltrándose para luchar, y también las organizaciones de la 'Resistencia' contrarrevolucionaria. Aunque se la apodaban de 'Lucha contra Bandidos', era, en realidad, una guerra bastante extensa, y no sólo en el lugar más conocido, la Sierra de Escambray del sur de Cuba, y también con bastante apoyo por parte de elementos campesinos opuestos al proceso revolucionario.

26. El internacionalismo era el nombre dado a la política de enviar a personal cubano a otros países del Tercer Mundo, en particular médicos, maestros, técnicos y militares. Aunque se inició a principios de los 60 con los primeros contactos con Argelia y con los varios grupos guerrille ros latinoamericanos, en realidad se desarrolló como política visible y declarada desde mediados de los 70. En los 80 llegaron hasta unos 40 los países tercermundistas que recibían esta asistencia humana de Cuba, la que también significaba para Cuba, no sólo un gran éxito publicitario en el Tercer Mundo, sino también una oportunidad nunca vista para miles de cubanos de viajar a otros países. El hecho de que la gran mayoría de estos eran también más pobres que Cuba servía de reforzar el compromiso a Cuba de estos 'internacionalistas'.

27. La guerra en Angola empezó en 1975, cuando el nuevo gobier no de Angola, el MPLA, pidió asistencia militar a sus aliados cubanos. Cuando terminó, en 1989, con la victoria clave de Cuito Carnavale, contra el ejército sudafricano - la que produjo el tratado de paz, la seguridad de Angola, la independencia de Namibia, y, hasta cierto punto, el inicio del proceso de negociación interna en Sudáfrica - habían luchado allí miles de cubanos (llegando hasta 40,000 a finales de los 70) 
Otra agrupación ahora más significativa en el sentido político que antes es la Asociación Nacional de Agricultores Pequeños (ANAP), organización que, a lo largo de los 60 y los 70 se veía frecuentemente como otro organismo paraestatal, dispuesto a mobilizar a los campesinos independientes para la obra de la Revolución ${ }^{28}$. Sin embargo, con la creación y la importancia futura de las UBPC -económica y políticamente- ya no se descarta la posibilidad de que esta organización coja una nueva vida, con cierta autonomía y una distancia creada con respecto al Partido, defendiendo los intereses particulares de los agricultores 'nuevos' y abogando por unas reformas comerciales, y no sólo económicas, sobre la base de una legitimidad 'diferente' 29 .

Desde luego, dada la naturaleza del sistema cubano, puede que estas organizaciones medioautónomas no sean tan independientes como aquí se sostiene, o, más probable, que vayan perdiendo la autonomía que actualmente parecen poseer, pero la verdad es que desde que se prognosticaba, en 1994, la emergencia de alguna clase de frente amplio de 'unidad nacional', para responder a la crisis política (incluyendo tal 'frente' a los CDR, la CTC y las FAR), la importancia de los tres organismos principales ha crecido notablemente en lugar de disminuirse ${ }^{30}$.

Pero, si se sigue sosteniendo la posibilidad de tal 'frente', de tal proceso de 'ampliación política', es imprescendible recordar a tres actores políticos más, tal vez -por ser más evidentemente independientes- más convencionalmente aceptables para la idea de una coalición de esta forma.

El primer 'actor' de este tipo debe ser esa parte de la comunidad emigrada que, desde 1990 , se ha manifestado más dispuesta a entrar en un proceso de diálogo con La Habana. Por cierto, es evidente, que, a partir de 1994, el liderazgo cubano viene realizando una política dirigida concretamente hacia ciertos sectores de esa comunidad, ya no tan monolítica como antes -aprovechando las diferencias generacionales, los intereses comerciales en Florida que buscan una entrada en Cuba, las redes familiares para asistir a los parientes necesitados de ayuda, o las ambiciones personales de los que buscaban un espacio político en la comunidad, fuera de la sombra del poderoso pero ya enfermo Jorge Mas Canosa. Las conferencias 'Nación y Emigración', celebradas en 1995, 1996 y 1997 no han sido los fracasos predecidos por la CANF (Cuban American National Foundation) ${ }^{31}$, y parece posible que la agresividad notable en ciertos sectores de la estructura política de la comunidad -en particular las actividades en 1996 de los llamados Hermanos al Rescate, de los que pusieron bombas en La Habana en 1997, y de los que organizaron las flotillas de rescate desde 1994- reflejen una batalla por la hegemonía interna de la comunidad, así como intentos por parte de ciertas agrupaciones de provocar a Castro para asegurar el compromiso renovado de un gobierno norteamericano algo indiferente a la causa de los exiliados. Evidentemente las decisiones de Clinton de firmar, primero, la Ley de Democracia Cubana (Cuba Democracy Act) de 1992, y, luego, la nefasta Ley Helms-Burton, en 1996 confirman el éxito de tal estrategia, ya que, en 1992, pare- cía muy posible que los demócratas alrededor de Clinton abogaban por una coexistencia más positiva con Castro. Por lo tanto, la posibilidad (intimada tentadoramente por Ricardo Alarcón en 1993) de extender la mano de amistad de un modo formal a elementos de la comunidad, tal vez para ofrecerles alguna participación limitada en el sistema político interno, permanece todavía vigente. En realidad, la crisis provocada en la comunidad por la visita del Papa en enero de 1998 ya está convirtiendo esta posibilidad en probabilidad, ya que la oposición fuerte por la CANF a cualquier participación de los emigrados en este evento fue rechazada tanto por la Iglesia como por miles de cubanos residentes en Florida, y muchos políticos de la comunidad aprovecharon la escisión para ofrecerse como alternativos al autoritario Mas Canosa. Ahora, con la muerte de éste, la unidad frágil de la comunidad, en términos políticos, se corrompe visiblemente.

El segundo actor evidente es la misma Iglesia católica. Otra vez, la evidencia reciente parece confirmar las tendencias vistas a principios de los 90, porque, hasta 1993, se veía todavía la continuación de todo un proceso de diálogo entre Iglesia y estado, un proceso iniciado en plena crisis de la iglesia en los 60, fomentado luego por el Vaticano y elaborado abiertamente a lo largo de los $80^{32}$. No obstante, en 1993, se vio el fin de esta política paciente de coexistencia, cuando la jerarquía se alejó pública y inesperadamente de la Revolución, criticando muchos aspectos del sistema y, evidentemente, intentando distanciarse de lo que les pare-

28. A partir de 1963 , aproximadamente un $30 \%$ de la tierra cultivada quedaba en manos de tales agricultores privados, dejados fuera del alcance directo del estado más típicamente por la importancia económica de sus cultivos (el café y el tabaco, en particular) y por el terreno difícil donde los cultivaban, dificultando las economías de escala mediante de las grandes granjas del pueblo. En los 70, una gran parte de la producción de los cítricos también se cultivaba por estos agricultores.

29. Las UBPC (Unidades Básicas de Producción Campesina) fueron creados en 1993 como parte principal de la reforma agraria que tuvo como objeto el incremento de la producción agrícola. Fundamentalmente las UBPC son las antiguas granjas estatales convertidas en cooperativas con cierta autonomía productiva y administrativa.

30. Véase Kapcia, 'Political Change in Cuba: Before and After the Exodus' (University of London Institute of Latin American Studies, Occasional Papers $\left.\mathrm{N}^{\circ} 9,1995\right)$, páginas 23-27.

31. Es esta la organización principal de la política anticastrista de la comunidad en Miami, dirigida desde los 80 por Mas Canosa, veterano de Playa Girón.

32. La historia de las relaciones entre la Iglesia y la revolución es compleja y no cabe dentro de las perspectivas de estado comunistas (como en La Europa Oriental). Primero, hay que recordar que, antes de 1959, la Iglesia fue tal vez la menos poderosa de toda Latinoamérica, socialmente marginada por la historia de la esclavitud, por las influencias norteamericanas ( $\mathrm{y}$ a veces evangélicas o bautistas) y por la asociación evidente con la clase media habanera blanca, servida por sacerdotes españoles muchas veces de tendencia política derechista. Cuando, en 1961, la jerarquía de la Iglesia se oponía a la que era una revolución popular (en parte porque la reforma educativa le privaba de su papel tradicional en las escuelas), y cuando unos sacerdotes participaron en la invasión, la expul sión de centenares de religiosos extranjeros era inevitable y popular Desde aquel momento, inició el Vaticano una política de diálogo con Castro y mejoraron las relaciones, asistadas por la emergencia en el continente de la Teología de la Liberación (la que le permitía a la iglesia a entrar en la perspectiva de la izquierda, animando también a muchos católicos radicales de otros países a persuadir a sus homólogos cubanos que participen ellos en esta ola de redefinición). En 1986, el proceso se vio claramente cuando el Partido Comunista abrió una Oficina de Asuntos Religiosos, dándole a un miembro destacado del Comité Central la reponsabilidad de dialogar con las diferentes iglesias. 
cía un sistema en declinación y sin apoyo. De eso resultó una especie de guerrita fría, durante la cual la Revolución marginó a los católicos más que a otros creyentes. Sin embargo, era evidente en aquel entonces que la postura crítica -resultado de una mala interpretación por parte de la jerarquía católica de las perspectivas para el sistema, creyendo la retórica de los emigrados acerca de la imagen de un régimen a punto de caer- no podía durar. A la Revolución le hacía falta una buena relación con la Iglesia, en ese momento de crisis y disagregación social, mientras que, como siempre en su historia, la Iglesia viviría más cómoda y más provechosamente en un contexto de convivencia y adaptación.

Hay tres factores que han afectado esta aproximación reciente. En primer lugar, se ha visto mucha presión por parte de las iglesias protestantes, que siempre han tenido una afinidad más íntima con el proceso revolucionario y, por consiguiente, han gozado de una situación más privilegiada. Estas iglesias, durante esta crisis, tenían gran miedo de que la división 'ideológica' entre la revolución y la religión pudiera provocar, o formentar o acelerar, una desintegración social peligrosa, y, ya que todavía compartían las metas sociales y morales del proceso revolucionario, trataban de mediar. El segundo factor ha sido la comprensión por parte de la jerarquía de que se habían equivocado, juzgando mal la situación interna y la fuerza y capacidad de sobrevivir del sistema. En esto les convencían más que nada todo el episodio de los balseros de agosto de 1994, el que les indicaba tanto los peligros sociales inherentes en la crisis como la fuerza del respaldo popular al sistema. El tercer factor ha sido la presión ejercida otra vez por el Vaticano, tratando de contruir puentes diplomáticos y ofreciéndose como mediador entre los miembros de 'la familia cubana', o sea viendo la oportunidad de un papel al estilo chileno.

De esto resultaron, primero, varias medidas tanto por la Iglesia cubana como por el liderazgo político diseñadas para reiniciar el diálogo y, luego, la invitación al Papa para enero de 1998. Era claro que tal visita tenía, objetivamente, la posibilidad de convertirse o en gran peligro para el sistema o en victoria publicitaria. Dado el anticomunismo visceral del pontífice polaco y su capacidad de lanzar retos políticos en los países que visita, era una jugada de posibles costes altos. Al contrario, dada la escasez de apoyo popular a la iglesia, dada la la evidencia de un respaldo todavía vigente a la Revolución y dadas las críticas ya abiertas que había expresado el Papa al bloqueo norteamericano, era también una visita con posibles beneficios enormes. Además, Fidel Castro entendía perfectamente que tanto al Papa como a la jerarquía cubana les preocupaban mucho los efectos sociales en Cuba de la crisis y de las reformas económicas (en particular la desigualdad creciente provocada y fomentada ahora por el turismo), tanto como le preocupaba a él, entendía bien que el mundo moral de él tenía muchos paralelos con las preocupaciones del Papa de 1998 (más que las preocupaciones políticas manifestadas en Polonia en 1981). Así, equilibrando los costes de una actuación opositora y crítica por el Papa contra los beneficios ciertos de unas críticas fuertes a los EEUU por el blo- queo así como la posibilidad de que alabara las ganacias sociales y los impulsos morales del proceso revolucionario, decidió seguir con la invitación. Tal como resultó, fue la visita un éxito enorme para Castro, dividiendo la unidad de los emigrados, dándole una oportunidad de extender la mano a unos líderes en Miami, organizando la acogida popular al Papa como manifestación nacionalista, aparentando como político dialoguero internamente, viéndole al Papa criticar a Washington y con el único coste político de una críticas templadas de la falta de respecto a los derechos humanos y una petición -ya esperada- de liberar a unos presos opositores. Accediendo a esta petición, Castro logró ganar mucho más de lo que haya perdido. Así, el proyecto antiguo de una alianza moral entre la Revolución y la Iglesia vuelve a ser vigente, y la Iglesia ya se convierte en mecanismo importante para facilitar el diálogo con los emigrados y con otros elementos disidentes dentro de Cuba.

Esta oposición interna es el tercer actor en este contexto. De los tres, es -y siempre ha sido- el más débil, dividida esta oposición entre grupúsculos efímeros o personalistas y minada permanentemente por la emigración de los disidentes. Por lo tanto, esta debilidad les ofrece a los protagonistas opositores la motivación de aceptar cualquier medida de diálogo que ofrezca el sistema. Igualmente, siendo estos grupos bastante débiles, y, así, no amenazando ellos al sistema, el diálogo con ellos le ofrece a la vanguardia revolucionaria la posibilidad de responder a las presiones externas -en particular de las sucesivas Cumbres Iberoamericanas, cuya retórica puede estar satisfecha con algún indicio de diálogo interno- pero sin peligro de repetir los errores de Gorbachev. Así es posible interpretar las aproximaciones hacia grupos disidentes como pasos de una política significativa de un proceso dialoguero: las reuniones entre Roberto Robaina y tres líderes opositores en Madrid en septiembre de 1994, las invitaciones a Eloy Gutiérrez Menoyo (de Concilio Cubano) de participar en seminarios sobre la representación política en junio de 1995. La suspensión aparente de este proceso en febrero de 1996 (con el incidente de las avionetas), cuando se prohibió una asamblea de Concilio Cubano y se detuvo a unos disidentes, no paró el proceso, el que se reinició con la visita del Papa. Por consiguiente, sigue muy posible la cooptación de ciertos grupos y ciertos líderes de la llamada disidencia, en particular porque a esta todavía le falta el apoyo popular. La prominencia y el tono reconciliador de Gutiérrez Menoyo en Miami durante los debates sobre la visita del Papa indican su deseo de aspirar no sólo a la hegemonía política dentro de la comunidad emigrada sino también de crear en Cuba alguna forma de movimiento de índole cristianodemócrata, actuando de 'oposición leal' vinculada con una Iglesia que siga dialogando con la Revolución.

A fin de cuentas, entonces, parece que, aunque no quepa dentro de las perspectivas convencionales, es bien posible distinguir la realidad de una 'reforma política', o, mejor dicho, de una 'evolución’ política. Es verdad que no ha sido rápida, a veces no bastante rápida para satisfacer las expectativas de comentaristas o políticos externos e incluso para muchos cubanos, y tampoco ha sido muy profun- 
da. No se habla aquí de 'ruptura' con el pasado, sino de adaptación cautelosa a las nuevas realidades.

Este ritmo tan lento se atribuye a tres factores. Primero, se atribuye al reconocimiento por parte del liderazgo de que ha sido más urgente, y más reivindicada, la necesidad de la reforma económica, haciendo que sea más importante en cuanto a los cambios políticos asegurar que estos no se produzcan paralelos al ritmo tan destabilizador y desmoralizante del proceso de la reforma económica. Así uno de los imperativos para el liderazgo ha sido asegurar que cualquier proceso de cambio político sea acompasado, escalonado, dirigido y cauteloso. La realidad es ésta -en una Cuba donde se han visto desaparecer tantas certidumbres, tal ritmo ha sido bastante aceptable para bastantes cubanos, en busca tanto de la seguridad como del cambio o de la reforma, para mantener la legitimidad.

A mediados de los 1990, era posible estimar, sobre la base de sondeos, votaciones y observaciones, que todavía quedaba leal, casi sin cuestionar, un $20-30 \%$ de la población, con un $40-50 \%$ tolerante pero quejándose ferozmente, pero más temoroso de las alternativas ${ }^{33}$. Si tales estimaciones eran correctas, y si siguen más o menos sin cambiar, pues la actitud del liderazgo - de ir más cautelosamente en cuanto a la 'reforma' política que en el campo económicoha sido apta.

El segundo factor para explicar el ritmo lento de las reformas políticas es la resistencia natural por parte de una vanguardia que sigue ya cuatro décadas timoneando, contra viento y marea, a la idea de cambiar la infraestructura política e ideológica del sistema. Puede atribuirse esta actitud a un conservadurismo innato de una vanguardia envejecida o a una determinación nacionalista a que sobreviva la Revolución según sus propios criterios, pero sigue siendo la verdad que tanto la vanguardia vieja como la generación nueva todavía se ven orientadas por parámetros de ideología que comprenden bien la necesidad de conservar la 'esencia' de la Revolución.

El tercer factor es la injerencia de presiones externas de Washington, de la Iglesia, de Madrid, de Bruselas. El deseo institucional de decelerar el ritmo del proceso de cambio se debe no sólo a la necesidad de asegurar la certidumbre política frente a la rapidez vertiginosa de las reformas económicas sino también a cierta tendencia inevitable de mantenerse en sus trece, frecuentemente con cierto apoyo popular. Es inevitable esta tendencia a atrincherarse porque, como deberían aprender los políticos de la Unión Europea, una realidad de la política cubana nunca comprendida por Washington es que cada presión ejercida desde fuera tiende a provocar una resistencia igual, tanto por una vanguardia acérrimamente nacionalista como por una parte suficiente de una población que lleva ya muchos años resistiendo 'el asedio' e interpretando como injerencia colonial o neocolonial cualquier intento de guiar los procesos internos. Según esta pauta, se entiende, por ejemplo la reacción de Castro a las críticas expuestas en la Cumbre Iberoamericana en julio de 1996 por Aznar, así como el incidente siguiente cuando los cubanos vetaron el nombramiento del nuevo embajador español, una reacción que tuvo una acogida bastante popular en Cuba. Hoy, como siempre en el caso del bloqueo americano, la presión externa suele ser contraproducente, obstaculizando el cambio más que acelerarlo.

Así, es claro que la cuestión de la 'reforma política' no está ni abierta ni cerrada. No está abierta, ya que todavía no existe ninguna intención, por parte del liderazgo y tampoco por la mayoría de la población, de buscar cambios fundamentales para el sistema; significa esto que la evolución seguirá por los caminos indicados. Sin embargo, tampoco está cerrada, ya que sigue evidente la capacidad del liderazgo de actuar flexiblemente y de sorprender, y ya que las presiones -externas al sistema político (en particular los efectos de las reformas económicas) o internas (expresadas por las varias formas de comunicación) - van a asegurar que siga adaptándose continuamente el sistema, y que cada ajuste tenga la capacidad de provocar otras medidas y otras presiones. En este sentido, sigue elaborándose el proceso largo de evolución.

Según esta pauta, está asegurado el futuro de la hegemonía del Partido, aunque puede que sobreviva esta dentro de una infraestructura política menos unipartidista que antes. Sin embargo es evidente que aquí no se habla del pluralismo multipartidario, sino de la expansión del mismo sistema para incluir e incluso cooptar a los grupos identificados, creando algún sistema de alianzas y cooptaciones y formalizando el proceso de 'diálogo nacional'. Dentro de tal infraestructura, es casi cierto que seguirán unos procesos ya iniciados: la creación gradual de una asamblea nacional permanente; la creación de un sistema más cooperativo en cuanto a la política, así como en la economía; una autonomía incrementada para grupos 'disidentes' favorecidos y a grupos selectos de la emigración dispuestos a dialogar; la retirada formal de Fidel Castro desde las decisiones diarias para convertirse en figura simbólica de gran importancia (presidente, determinante de política exterior, conciencia ideológica), reconociendo su valor legitimador para una parte significativa de la población pero, al mismo tiempo, la importancia de ofrecer una nueva cara a los jóvenes. En realidad se habla de la posibilidad real de que Fidel Castro siga convirtiéndose poco a poco en mascarón de proa, necesaria pero sin la influencia directa de los años pasados ${ }^{34}$.

En conclusión, ¿cuáles son las claves que van a determinar este futuro posible? Simplemente, las claves son las de siempre. En primer lugar, el factor más determinante serán los éxitos o fracasos de la economía, a nivel macro (afectada por Helms-Burton, minada por la falta de capitales, etcétera) y al nivel familiar, en la capacidad de la economía de darles a los cubanos su pan de cada día. Otro factor clave deben ser el nivel y la eficacia de las presiones ejercidas desde Washington, recordando siempre esa realidad de Cuba, de que cuanto menos se ejerce la presión

33. Véase Kapcia (1995), página 22.

34. En realidad, este proceso de retirada no es nuevo, ya que, en los 70, se inició, para pararse frente a la crisis desde 1986, pero es aún más evidente en muchos aspectos, y la institucionalización de las reformas económicas y la desaparición de una crisis potencial en la política hacen que pueda reiniciarse otra vez. 
tantas más posibilidades hay de una evolución rápida. Otro factor clave será la participación de Fidel Castro en la estructura y el proceso de determinar las decisiones, un factor capaz de actuar en dos direcciones diferentes -hacia un Castro comprometido a la reforma política (lo que daría el "imprimatur" ideológico a ella, así que las esperanzas de un cambio real pueden, paradójicamente, depender de su apoyo), o hacia un Castro determinado a resistir cualquier cambio, lo que puede retrasar el proceso y provocar antagonismos profundos. El cuarto factor será la eficacia de los sistemas de participación; en esto la revigoración de ciertos organismos puede indicar tanto una evolución continua como una resistencia al cambio, y es cierto que la ineficacia de tales órganos podría ser peligrosa para el orden social. Finalmente, es clave el papel de las FAR.

Lo interesante aquí es que estos factores sean precisamente los mismos que podrían haberse identificado en cualquier momento del proceso entero desde 1959, todo lo que confirma el argumento básico de este ensayo, o sea que más vale hablar de un proceso de evolución continua, adaptación y reflejo, que de un proceso de 'reforma', una perspectiva que no logra entrar en la complejidad de un sistema, una cultura política y todo un fenómeno político que sigue confundiendo a los que lo observan desde fuera.

\title{
RESUMEN
}

El autor trata de dar respuesta a la continua evolución de la estructura política cubana a partir de los cambios acaecidos en 1989 e incluso remontándose al mismo momento de la victoria de la revolución en 1959, enfatizando los debates y condicionantes internos. En este sentido, identifica los principales factores que pueden determinar el futuro de la Isla: 1) los éxitos o fracasos de la economía, a nivel macro pero también a nivel doméstico; 2) el nivel y la eficacia de las presiones ejercidas desde Washington; 3) la participación de Fidel Castro en la estructura y el proceso de determinar las decisiones; 4) la eficacia de los sistemas de participación; y 5) el papel a jugar por las Fuerzas Armadas de la Revolución (FAR).

Palabras claves: evolución política, estructura política, participación, FAR.

\begin{abstract}
This article analyzes the internal conditionants of the evolution of the Cuban political structure from the 1959 revolution. It identifies the main factors affecting the Cuban future: 1) the successes and failures of the economy; 2) the level and efficency of the pressures exercised by Washington; 3) the role of Fidel Castro in the decisionmaking process; 4)the efficiency of the participatory processes, and 5) the role played by the Armed Forces.
\end{abstract}

Key words: political evolution, political structure, participation. 